\title{
Different efficacy in the non-small cell lung cancer patient with bilateral synchronous lesions treated with neoadjuvant gefitinib therapy: a case report
}

\author{
Wenxing Du ${ }^{1}$, Yandong Zhao ${ }^{1}$, Yunpeng Xuan ${ }^{1}$, Yi Qin ${ }^{1}$, Rongjian Xu ${ }^{1}$, Balazs Halmos ${ }^{2}$, Thomas Fabian ${ }^{3}$, \\ Wenjie Jiao ${ }^{1}$ \\ ${ }^{1}$ Department of Thoracic Surgery, the Affiliated Hospital of Qingdao University, Qingdao 266071, China; ${ }^{2}$ Department of Oncology, Albert Einstein \\ College of Medicine/Montefiore Medical Center, Bronx, NY, USA; ${ }^{3}$ Department of Surgery, Albany Medical Center, Albany, NY, USA \\ Correspondence to: Wenjie Jiao. Department of Thoracic Surgery, the Affiliated Hospital of Qingdao University, Qingdao 266071, China. \\ Email: jiaowj@qduhospital.cn.
}

\begin{abstract}
Gefitinib, the first-generation epidermal growth factor receptor tyrosine kinase inhibitor (EGFRTKI), has become the standard of care for the first-line of therapy for advanced non-small cell lung cancer (NSCLC) with common EGFR mutation. However, the efficacy of preoperative gefitinib therapy in patients with common EGFR mutations remains poorly defined. We describe a NSCLC patient with bilateral synchronous lesions who had a significantly positive response to gefitinib before radical surgical resection. At the time of initial diagnosis, we were unable to confirm whether the two lesions were metastatic or synchronous primary lesions. Accordingly, we performed CT-guided percutaneous left lung biopsy resulting in a diagnosis of lung adenocarcinoma with exon 21 L858R point mutation of EGFR, This diagnosis was followed by preoperative gefitinib therapy for 8 weeks leading to a significant reduction in the lesion in the left lower lobe. Then the left lower lobectomy and mediastinal lymphadenectomy were performed. In addition, 3 months following resection of the left lower lobe tumor the patient underwent a right lower lobe wedge resection. This report indicates that NSCLC patient harboring common EGFR mutation accepting the first-generation EGFR-TKI gefitinib as a neoadjuvant targeted therapy option is safe, feasible, and welltolerated.
\end{abstract}

Keywords: Gefitinib; neoadjuvant targeted therapy; lung neoplasms

Submitted Oct 23, 2019. Accepted for publication Feb 20, 2020.

doi: $10.21037 /$ jtd.2020.02.60

View this article at: http://dx.doi.org/10.21037/jtd.2020.02.60

\section{Introduction}

Gefitinib, the first-generation epidermal growth factor receptor tyrosine kinase inhibitor (EGFR-TKI), has become the standard of care for the first-line of therapy for advanced non-small cell lung cancer (NSCLC) with common EGFR mutation $(1,2)$. Many studies have consistently demonstrated that common EGFR-mutated advanced NSCLC patients treated with EGFR-TKIs can achieve better survival outcomes than with conventional chemotherapy (3-6). However, its efficacy as a neoadjuvant targeted therapy in patients with EGFR mutation-positive NSCLC remains poorly defined.
Recently, we have encountered a case indicating that preoperative gefitinib therapy may work well. In this report, we describe the NSCLC patient harboring common EGFR mutation who had a significantly positive response to gefitinib before radical surgical resection.

\section{Case presentation}

A 42-year-old woman was admitted to our hospital with a pulmonary shadow. The thoracic computed tomography (CT) scan revealed two pulmonary lesions, 19.5 and $20.9 \mathrm{~mm}$ in size, which located in the posterior basal segment of the 

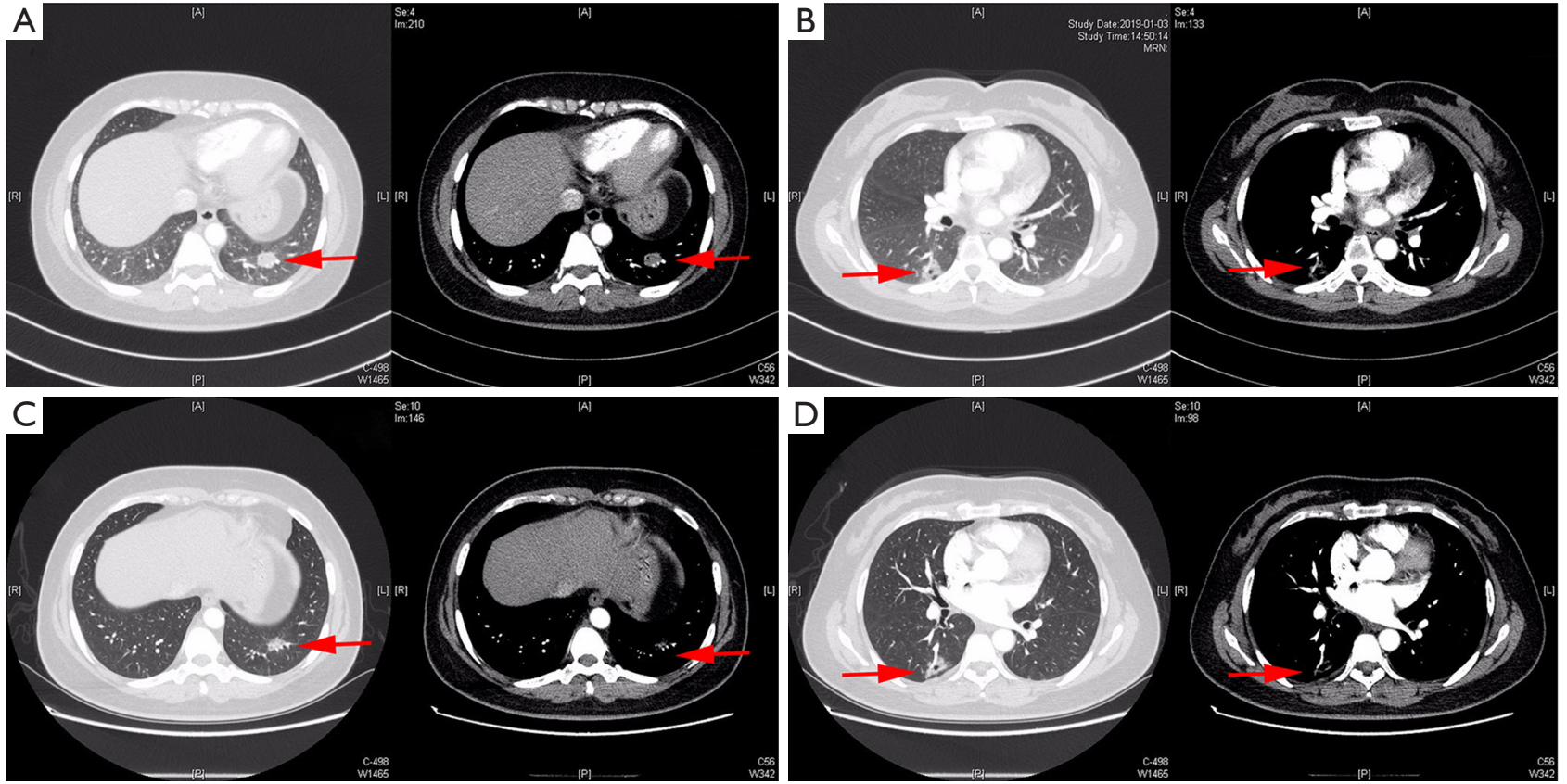

Figure 1 Thoracic computed tomography scan revealed the lesions. (A) The lesion in the left lower lobe before neoadjuvant gefitinib therapy; (B) the lesion in the right lower lobe before neoadjuvant gefitinib therapy; (C) the lesion in the left lower lobe after neoadjuvant gefitinib therapy; (D) the lesion in the right lower lobe after neoadjuvant gefitinib therapy. The red arrows mean location of the lesions.

left lower lobe (Figure 1A) and the superior segment of right lower lobe (Figure 1B) respectively, while no significant enlargement was observed in the mediastinal lymph nodes. Routine blood test showed the white blood cell count was $5.46 \times 10^{9} / \mathrm{L}$, which was normal, and the patient did not have any obvious positive symptoms or signs.

The patient chose CT-guided percutaneous left lung biopsy on January 9, 2019, and the pathological result was non-small cell lung adenocarcinoma with exon 21 L858R point mutation of EGFR. On January 15, 2019, positron emission tomography/computed tomography (PET/CT) examination findings exhibited that the bilateral lung lesions were highly likely to be tumors and no metastases were found in other sites. Then she was offered neoadjuvant gefitinib therapy which she received for 8 weeks. Subsequently, the thoracic CT scan had revealed that the lesion located in the left lower lobe reduced significantly (Figure 1C), about $13 \mathrm{~mm}$ in size, while the other lesion in the right lung showed no obvious change (Figure 1D).

This patient had the indications for surgery and accepted surgical operation treatment. Presurgical evaluations, including the pulmonary function test and metastatic examination, were normal. Then the left lower lobectomy and mediastinal lymphadenectomy were performed. In addition, 3 months following resection of the left lower lobe tumor the patient underwent a right lower lobe wedge resection.

\section{Surgical technique}

The left lower lobectomy and mediastinal lymphadenectomy were performed on April 2, 2019. The patient was placed in a right lateral decubitus position and induced by general anesthesia with double-lumen endotracheal intubation. The two ports had been created. The camera port was about $1.5 \mathrm{~cm}$ long locating in the seventh intercostal space at the midaxillary line and another port, $3 \mathrm{~cm}$ in size, locates in the fourth intercostal space at the anterior axillary line. The operator and the assistants stood at the abdominal and backside of the patient, respectively.

Intraoperative exploration showed that the lesion located in the left lower lobe without lymph nodes enlargement. Dissect the mediastinal pleura with the unipolar cautery hook, and the 4L, 5, 6, 7, 9, 10L, 11L station lymph nodes were resected. Expose the left inferior pulmonary ligament and dissociate the inferior pulmonary vein. Transect the vein by the endoscopic stapler. Then dissociate and cut open the left lower bronchus and inferior pulmonary artery. 
Finally, resect the fused pulmonary fissure and remove the specimen later. With an airway pressure of $25 \mathrm{cmH}_{2} \mathrm{O}$, no air leakage was found. The operation finished successfully.

\section{Clinic result}

Frozen section examination of resection margins was negative. The patient was discharged in the 5 th postoperative days without complications. No mortality or major morbidity present for 5 months after the operation. The final pathologic examination was invasive adenocarcinoma without lymph node involvement while the postoperative pathological result was ypT1cN0M0, stage I. After surgery, the patient continues to receive targeted therapy.

Furthermore, 3 months following resection of the left lower lobe tumor the patient underwent a right lower lobe wedge resection on July 10, 2019. The pathological result was invasive adenocarcinoma harboring KIF5B-RET fusion without EGFR mutation-positive, and the postoperative pathological result was ypT1bN0M0, stage I. No mortality or major morbidity presents.

\section{IMDT discussion}

\section{Department of Oncology}

The CT scan revealed bilateral pulmonary nodules in this patient. According to the images, the lesions presented lobulated sign, pleural indentation sign, and vessel convergence sign, and the mediastinal lymph nodes were not significantly enlarged. Combining the results of the interrogation and physical examination showed that the patient had no obvious positive symptoms or signs. Laboratory tests showed the white blood cell counts were normal. Based on the above, it was considered that the possibility of pulmonary inflammatory nodules, pulmonary tuberculosis, benign tumors of the lungs, or lymphoma was small, while pulmonary malignant tumors might be larger, and lung metastases could not be ruled out. Then the CT-guided biopsy confirmed that the lesion in the left lower lobe was NSCLC with common EGFR mutations. PET/CT examination findings exhibited that bilateral lesions were highly likely to be tumors and no metastases were found in other parts of the body. At this time, we mainly consider the following two diagnostic possibilities. One possibility is that both lung nodules are primary lung malignancies and the clinical stages of both tumors are stage
IA. It is recommended that the patient undergoes bilateral lung surgery, which can be performed simultaneously or in stages. Adjuvant treatment is optional after surgery. Another possibility is that the bilateral lesions are lung metastases and the clinical stage is IVA. The patient is recommended to be treated conservatively without operation. In any case, we believe that conservative treatment can be the preferred treatment, such as targeted drug therapy, chemotherapy, radiotherapy, or combination therapy. After a period of treatment, we will formulate the next diagnosis and treatment plan based on the efficacy.

\section{Department of Thoracic Surgery}

The first-generation EGFR-TKI gefitinib has been widely used in first-line treatment of advanced NSCLC with common EGFR mutation-positive patients $(1,2)$. However, the efficacy of preoperative gefitinib therapy in patients with common EGFR mutations remains poorly defined. In a study by Deng et al. (7), among patients with advanced NSCLC harboring common EGFR mutations, gefitinib demonstrated no survival benefit comparing with chemotherapy, but it is still the optimal choice for patients with common mutations. In this case, we were unable to confirm whether the two lesions were metastatic or synchronous primary lesions at the beginning. All we know was that the lesion of the left lower lobe was invasive lung adenocarcinoma with exon 21 L858R point mutation of EGFR. Then the patient chose preoperative gefitinib therapy for 8 weeks, and the lesion in the left lower lobe demonstrated significant reduction in size, while the other lesion in the right lung showed no obvious change. Until this time, we thought that the two lesions were likely to be bilateral synchronous primary lesions.

Moreover, the pathological examination of the lesion in the right lower lobe was lung adenocarcinoma with KIF5B-RET fusion. There was no significant change after treatment with gefitinib, indicating that the KIF5B-RET fusion is insensitive or resistant to first-generation EGFRTKIs. This suggests that when we choose first-generation EGFR-TKIs for preoperative targeted therapy, the support of genetic testing results remains to be necessary. And the diagnosis of this case was more likely to be bilateral synchronous primary lesions (8).

During the operation, no additional hydrothorax, tissue adherence, and prone to bleeding were observed. It can be speculated that preoperative gefitinib therapy can improve the radical resection rate of not only resectable 
NCSLC patients but also unresectable NCSLC patients with common EGFR mutations (9). In a word, neoadjuvant gefitinib therapy was safe, feasible, and well-tolerated.

In this case, there are a few questions that should be further explored.

\section{Question 1: How to better diagnose multiple bilateral lesions in multiple primary lung cancer or metastasis?}

\section{Expert opinion 1: Dr. Balazs Halmos}

Patients presenting with multiple lesions is a fairly common issue the clinician faces, especially amongst smokers as a result of field cancerization (10-14). While distinguishing metastatic disease from a synchronous primary can be a great challenge, there are several possible ways to assess this, such as imaging features that might suggest a primary lesion (typically speculation represents a primary lesion) versus metastatic lesion (many times well circumscribed lesion without associated ground glass features or spiculation). Next, if pathological specimen can be obtained from both lesions, pathological examination can demonstrate histological subtype differences or differences in grade/ differentiation/histological patterns that might suggest a synchronous primary. Lastly, nowadays molecular testing/ molecular fingerprinting can add another layer of evidence arguing for one or another (14). Traditionally, if despite best efforts we cannot ascertain histological derivation confidently, the benefit of the doubt is given when there could be curative treatment offered in case of synchronous primaries—such as the bilateral operations appropriately performed in this interesting case report.

\section{Expert opinion 2: Dr. Thomas Fabian}

Accurately staging malignancy is critical in any cancer. Failure to do so invariably results in over or under treatment of the malignancy. Certain tumors are easier to stage than others and perhaps the hardest is Synchronous Multiple Primary Lung Cancers (SMPLC). Inaccurately staging these patients as metastatic results in poor outcomes and typically death. Whereas accurately staging offers these patients the opportunity of cure. Some of these challenges are exacerbated by our own perceived notions of lung cancers propensity to metastasize to the lung. However, this perspective has been challenged more recently and data increasingly shows that frequently what is considered metastatic disease of the lung to the lung is actually SMPLC. Chen et al. published 39 patients undergoing resection of multiple tumors (15). In those 39 patients multiple tumors were tested for mutations and $94.6 \%$ discordance among driver mutations was found.

This case report demonstrates a similarity to Chen's publication both tumors had discordant driver mutations. The variable response rate between the left and right side tumors (both histologic Adenocarcinoma) presented here is consistent with in SMPLC and the different responses are directly attributable to the actionable mutation. Effectively, the neoadjuvant treatment with Gefitinib confirmed separate primaries, perhaps inadvertently. Clinical attentiveness to the discrepancy in tumor response ultimately led this patient to the appropriate treatment which was bilateral pulmonary resections.

Question 2: When it is not possible to determine whether multiple primary lung cancer or contralateral metastasis of lung cancer, can an EGFR-TKI therapy be a preoperative diagnostic treatment method?

\section{Expert opinion 1: Dr. Balazs Halmos}

As highlighted above, the traditional approach is aggressive management with definitive therapy to the bilateral lesionsmany times surgery with/without radiation. It is intriguing to consider whether neoadjuvant therapy and differential responses to such could provide guidance-in this case certainly at least suggestive evidence was gained by the short neoadjuvant treatment course which the subject tolerated well.

\section{Expert opinion 2: Dr. Thomas Fabian}

Perhaps in patients with more confusing clinical pictures neoadjuvant therapy based on actionable mutations can be one tool to help separate SMPLC from metastatic disease.

Question 3: What is the application prospect of EGFR-TKI therapy as preoperative adjuvant therapy for unresectable advanced non-small cell lung cancer?

\section{Expert opinion 1: Dr. Balazs Halmos}

Neoadjuvant EGFR-targeted therapy is actively being investigated as an effective and safe approach and available data certainly demonstrate a high chance of response and good tolerance (16). Whether ultimately overall survival might improve as a result of neoadjuvant or adjuvant targeted therapy and whether neoadjuvant therapy could render borderline resectable tumors resectable remains important research questions. 
Expert opinion 2: Dr. Thomas Fabian

EGFR-TKI as neoadjuvant therapy is interesting and evolving treatment option, at what point in the future it may compliment or replace traditional neoadjuvant chemotherapy or neoadjuvant chemoradiation therapy is anybody's guess. Certainly the tumor response for some of these patients is remarkable and in unique situations we are already finding ourselves offering surgical resection for previously unresectable disease. Results and outcomes of these cases are not widely known and remain mostly antidotal.

\section{Conclusions}

This report indicated that NSCLC patient harboring common EGFR mutation accepting the first-generation EGFR-TKI gefitinib as a neoadjuvant targeted therapy option was safe, feasible, and well-tolerated.

\section{Acknowledgments}

Funding: None.

\section{Footnote}

Conflicts of Interest: All authors have completed the ICMJE uniform disclosure form (available at http://dx.doi. org/10.21037/jtd.2020.02.60). The authors have no conflicts of interest to declare.

Ethical Statement: The authors are accountable for all aspects of the work in ensuring that questions related to the accuracy or integrity of any part of the work are appropriately investigated and resolved. Written informed consent was obtained from the patient for publication of this case report and any accompanying images.

Open Access Statement: This is an Open Access article distributed in accordance with the Creative Commons Attribution-NonCommercial-NoDerivs 4.0 International License (CC BY-NC-ND 4.0), which permits the noncommercial replication and distribution of the article with the strict proviso that no changes or edits are made and the original work is properly cited (including links to both the formal publication through the relevant DOI and the license). See: https://creativecommons.org/licenses/by-nc-nd/4.0/.

\section{References}

1. Novello S, Barlesi F, Califano R, et al. Metastatic nonsmall-cell lung cancer: ESMO Clinical Practice Guidelines for diagnosis, treatment and follow-up. Ann Oncol 2016;27:v1-v27.

2. Ma Y, Xin S, Lin Q, et al. The analysis of pharmacokinetic and pharmacogenomic impact on gefitinib efficacy in advanced non-small cell lung cancer patients: results from a prospective cohort study. Ann Transl Med 2019;7:806.

3. Yang JC, Wu YL, Chan V, et al. Epidermal growth factor receptor mutation analysis in previously unanalyzed histology samples and cytology samples from the phase III Iressa Pan-ASia Study (IPASS). Lung Cancer 2014;83:174-81.

4. Wang Q, Shen B, Qin X, et al. Akt/mTOR and AMPK signaling pathways are responsible for liver $\mathrm{X}$ receptor agonist GW3965-enhanced gefitinib sensitivity in nonsmall cell lung cancer cell lines. Transl Cancer Res 2019;8:66-76.

5. Zhou C, Wu YL, Chen G, et al. Final overall survival results from a randomised, phase III study of erlotinib versus chemotherapy as first-line treatment of EGFR mutation-positive advanced non-small-cell lung cancer (OPTIMAL, CTONG-0802). Ann Oncol 2015;26:1877-83.

6. Mok TS, Cheng Y, Zhou X, et al. Improvement in Overall Survival in a Randomized Study That Compared Dacomitinib With Gefitinib in Patients With Advanced Non-Small-Cell Lung Cancer and EGFR-Activating Mutations. J Clin Oncol 2018;36:2244-50.

7. Deng W, Lei Y, Liu S, et al. Comparing overall survival between first generation EGFR-TKIs and chemotherapy in lung cancer patients with Del19/L858R. Chin J Cancer Res 2016;28:339-47.

8. Guo H, Shen-Tu Y. Research Progress in Diagnosis and Management Strategies of Multiple Primary Lung Cancer. Zhongguo Fei Ai Za Zhi 2016;19:307-11.

9. Wang T, Liu Y, Zhou B, et al. Retrospective analysis of icotinib neoadjuvant therapy of 63 lung cancer patients. Indian J Cancer 2017;54:215-22.

10. Vignot S, Frampton GM, Soria JC, et al. Next-generation sequencing reveals high concordance of recurrent somatic alterations between primary tumor and metastases from patients with non-small-cell lung cancer. J Clin Oncol 2013;31:2167-72. 
11. Stiles BM. Say goodbye to Martini and Melamed: genomic classification of multiple synchronous lung cancer. J Thorac Dis 2017;9:E87-E88.

12. Murphy SJ, Aubry MC, Harris FR, et al. Identification of independent primary tumors and intrapulmonary metastases using DNA rearrangements in non-small-cell lung cancer. J Clin Oncol 2014;32:4050-8.

13. Liu Y, Zhang J, Li L, et al. Genomic heterogeneity of multiple synchronous lung cancer. Nat Commun 2016;7:13200.

14. Girard N, Ostrovnaya I, Lau C, et al. Genomic and mutational profiling to assess clonal relationships between

Cite this article as: Du W, Zhao Y, Xuan Y, Qin Y, Xu R, Halmos B, Fabian T, Jiao W. Different efficacy in the nonsmall cell lung cancer patient with bilateral synchronous lesions treated with neoadjuvant gefitinib therapy: a case report. J Thorac Dis 2020;12(4):1582-1587. doi: 10.21037/jtd.2020.02.60 multiple non-small cell lung cancers. Clin Cancer Res 2009;15:5184-90.

15. Chen K, Chen W, Cai J, et al. Favorable prognosis and high discrepancy of genetic features in surgical patients with multiple primary lung cancers. J Thorac Cardiovasc Surg 2018;155:371-379.e1.

16. Chang JC, Alex D, Bott M, et al. Comprehensive NextGeneration Sequencing Unambiguously Distinguishes Separate Primary Lung Carcinomas From Intrapulmonary Metastases: Comparison with Standard Histopathologic Approach. Clin Cancer Res 2019;25:7113-25. 